\title{
A method of estimating the p-adic sizes of common zeros of partial derivative polynomials associated with a complete cubic form
}

\begin{abstract}
Let $\mathrm{x}=(\mathrm{x} 1, \mathrm{x} 2$,é, $\mathrm{xn})$ be a vector in the space $\mathrm{Q} \mathrm{n}$ with $\mathrm{Q}$ field of rational numbers and $\mathrm{q}$ be a positive integer, $\mathrm{f}$ a polynomial in $\mathrm{x}$ with coefficient in $\mathrm{Q}$. The exponential sum associated with $\mathrm{f}$ is defined as $\mathrm{S}(\mathrm{f} ; \mathrm{q})=\mathbb{E x m o d q e}((2 \mathrm{if}(\mathrm{x})) / \mathrm{q})$, where the sum is taken over a complete set of residues modulo $q$. The value of $S(f ; q)$ depends on the estimate of cardinality $|V|$, the number of elements contained in the set $\mathrm{V}=\{\mathrm{xmodq} \mid \mathrm{fx} f 0$ modq $\}$ where $\mathrm{fx}$ is the partial derivative of $\mathrm{f}$ with respect to $\mathrm{x}$. To determine the cardinality of $\mathrm{V}$, the p-adic sizes of common zeros of the partial derivative polynomials need to be obtained. In this paper, we estimate the $p$-adic sizes of common zeros of partial derivative polynomials of $f(x, y)$ in $\mathrm{Qp}[\mathrm{x}, \mathrm{y}]$ with a complete cubic form by using Newton polyhedron technique. The polynomial is of the form $f(x, y)=a x 3+b x 2 y+c x y 2+d y 3+32 a x 2+b x y+12 c y 2+s x+t y+k$.
\end{abstract}

Keyword: Exponential sums; Cardinality; P-adic sizes; Newton polyhedron 